\title{
Consistent delayed unilateral neuronal death after modified transient focal cerebral ischemia in mice that mimics neuronal injury after transient global cerebral ischemia
}

\author{
Yasuo Nishijima, MD, PhD, Kuniyasu Niizuma, MD, PhD, Miki Fujimura, MD, PhD, \\ Yosuke Akamatsu, MD, Hiroaki Shimizu, MD, PhD, and Teiji Tominaga, MD, PhD \\ Department of Neurosurgery, Tohoku University Graduate School of Medicine, Sendai, Japan
}

\begin{abstract}
OBJECT Numerous studies have attempted to reveal the pathophysiology of ischemic neuronal injury using a representative transient global cerebral ischemia (tGCl) model in rodents; however, most of them have used gerbil or rat models. Recent advances in transgene and gene-knockout technology have enabled the precise molecular mechanisms of ischemic brain injury to be investigated. Because the predominant species for the study of genetic mutations is the mouse, a representative mouse model of $\mathrm{tGCl}$ is of particular importance. However, simple mouse models of $\mathrm{tGCl}$ are less reproducible; therefore, a more complex process or longer duration of ischemia, which causes a high mortality rate, has been used in previous tGCl models in mice. In this study, the authors aimed to overcome these problems and attempted to produce consistent unilateral delayed hippocampal CA1 neuronal death in mice.
\end{abstract}

METHODS C57BL/6 mice were subjected to short-term unilateral cerebral ischemia using a 4-mm silicone-coated intraluminal suture to obstruct the origin of the posterior cerebral artery (PCA), and regional cerebral blood flow (rCBF) of the PCA territory was measured using laser speckle flowmetry. The mice were randomly assigned to groups of different ischemic durations and histologically evaluated at different time points after ischemia. The survival rate and neurological score of the group that experienced 15 minutes of ischemia were also evaluated.

RESULTS Consistent neuronal death was observed in the medial CA1 subregion 4 days after 15 minutes of ischemia in the group of mice with a reduction in $\mathrm{rCBF}$ of $<65 \%$ in the PCA territory during ischemia. Morphologically degenerated cells were mostly positive for terminal deoxynucleotidyl transferase-mediated deoxyuridine triphosphate nick-end labeling and cleaved caspase 3 staining 4 days after ischemia. The survival rates of the mice 24 hours $(n=24), 4$ days $(n=$ $15)$, and 7 days $(n=7)$ after being subjected to 15 minutes of ischemia were $95.8 \%, 100 \%$, and $100 \%$, respectively, and the mice had slight motor deficits.

CONCLUSIONS The authors established a model of delayed unilateral hippocampal neuronal death in C57BL/6 mice by inducing ischemia in the PCA territory using an intraluminal suture method and established inclusion criteria for PCAterritory $\mathrm{rCBF}$ monitored by laser speckle flowmetry. This model may be useful for investigating the precise molecular mechanisms of ischemic brain injury.

http://thejns.org/doi/abs/10.3171/2014.9.JNS14778

KEY WORDS apoptosis; delayed neuronal death; hippocampus; laser speckle flowmetry; mice; unilateral

$\mathrm{T}$ RANSIENT global cerebral ischemia (tGCI) has been shown to cause delayed cell death in several vulnerable regions of the brain, including the hippocampal CA1 subregion. ${ }^{10,19,21}$ Animal models of tGCI have been widely used to analyze the mechanisms of ischemic neuronal injury.,17,18,21 Although the results of numerous studies have suggested that various factors participate in the process of delayed cell death, ${ }^{12}$ the exact mechanisms remain unclear.

Recent advances in transgene and gene-knockout tech-

ABBREVIATIONS BCCAO = bilateral CCA occlusion; $\mathrm{CBF}=$ cerebral blood flow; $\mathrm{CCA}=$ common carotid artery; $\mathrm{DAB}=$ diaminobenzidine; $\mathrm{ECA}=$ external carotid artery; $I C A=$ internal carotid artery; $L S F=$ laser speckle flowmetry; $M C A=$ middle cerebral artery; $M C A O=M C A$ occlusion; $P B S=$ phosphate-buffered saline; $P C A=$ posterior cerebral artery; $\mathrm{PCOA}=$ posterior communicating artery; $\mathrm{rCBF}=$ regional $\mathrm{CBF} ; \mathrm{ROI}=$ region of interest; $\mathrm{tGCI}=$ transient global cerebral ischemia; $\mathrm{TTC}=2,3,5$-triphenyltetrazolium chloride; TUNEL = terminal deoxynucleotidyl transferase-mediated deoxyuridine triphosphate nick-end labeling.

SUBMITTED April 7, 2014. ACCEPTED September 10, 2014.

INCLUDE WHEN CITING Published online February 27, 2015; DOI: 10.3171/2014.9.JNS14778.

DISCLOSURE The authors report no conflict of interest concerning the materials or methods used in this study or the findings specified in this paper. The following grant funded this study: JSPS KAKENHI grant no. 24659642. 
nologies have enabled the precise molecular mechanisms of ischemic brain injury to be investigated. Because the predominant species for studying genetic mutations is the mouse, a representative mouse model of tGCI is of particular importance. Although mouse tGCI models that produce hippocampal CA1 injury have been established over the past 20 years, ${ }^{6,13,14,16,20,25-29}$ complicated procedures or a high mortality rate may limit their application. Longer ischemic durations or stronger reductions in hippocampal blood flow are needed to ensure consistent injury; however, both of these factors have been associated with high mortality rates. Moreover, individual differences in collateral blood flow, especially that from the posterior communicating artery (PCoA), cause inconsistent hippocampal neuronal injury. ${ }^{6,11,20,25}$

The main feeding artery to the hippocampus is the longitudinal hippocampal artery, which diverges from the distal part of the posterior cerebral artery (PCA) after convergence of the PCoA. ${ }^{4}$ Occlusion of the PCA, PCoA, and collateral circulation to the distal part of the PCA is known to induce hippocampal ischemia. Although previous models used bilateral forebrain ischemia to induce hippocampal ischemia, stronger ischemic insults caused high mortality rates, whereas weaker ischemic insults resulted in inconsistent hippocampal injury. Thus, we hypothesized that more selective unilateral hippocampal ischemia could significantly contribute to consistent hippocampal injury with low mortality rates.

The purpose of this study was to establish a mouse model of consistent degrees of hippocampal CA1 neuronal death with lower mortality rates. To induce unilateral hippocampal ischemia, we created a modified middle cerebral artery occlusion (MCAO) model using an intraluminal suture in C57BL/6 mice, which are commonly used in the production of transgenic animals ${ }^{9}$ and have less-patent PCoAs. ${ }^{11}$ Unilateral hippocampal ischemia was induced in mice by using a 4-mm silicone-coated intraluminal suture that occluded the orifice of the PCA. Regional cerebral blood flow (rCBF) of the PCA territory was topographically monitored using laser speckle flowmetry (LSF) to exclude mice with well-developed collateral blood flow through the PCoA on the ischemic side. We optimized the duration of ischemia that did not induce infarctions of the MCA territory but caused hippocampal neuronal death. We histologically examined delayed neuronal cell death after unilateral hippocampal ischemia in these C57BL/6 mice. Moreover, we evaluated the physiological parameters and neurological symptoms after unilateral hippocampal ischemia.

\section{Methods}

\section{Animal Preparation}

All procedures were approved by the animal care facility of Tohoku University Graduate School of Medicine. Male C57BL/6 mice (Japan SLC, Inc.) aged 8-12 weeks (weighing 20-26 g) at the time of surgery were used in this study.

\section{Unilateral Hippocampal Ischemia}

A 4-mm silicone-coated suture, $210 \mu \mathrm{m}$ in diameter, was considered to be sufficient in length and thickness to occlude the origin of the PCA when it was inserted $9 \mathrm{~mm}$ from the common carotid artery (CCA) bifurcation. ${ }^{1,22,23}$ Our group previously demonstrated that 120 minutes of ischemia using a 4-mm silicone-coated intraluminal suture induced cerebral infarctions in the PCA territory including the thalamus, hippocampus, and posterior part of the cerebral cortex in mice with a hypoplastic PCoA. ${ }^{1}$ This finding showed that the 4-mm suture seemed sufficient to obstruct the origin of the PCA and induce unilateral hippocampal ischemia.

Each mouse was anesthetized with a ketamine-xylazine mixture $(40 \mathrm{mg} / \mathrm{kg}$ ketamine and $4 \mathrm{mg} / \mathrm{kg}$ xylazine, total volume of injection $1.0 \mathrm{ml} / \mathrm{kg}$ intraperitoneally) and breathed room air spontaneously. Rectal temperature during all surgical procedures was maintained at $36.0^{\circ} \mathrm{C}-37.5^{\circ} \mathrm{C}$ using a feedback-regulated heating pad (BWT-100; Bio Research Center Co., Ltd.). Arterial blood pressure was measured using the tail-cuff auto-pickup method (Softron Co., Ltd.). With the mouse in the prone position and fixed in a head holder (SG-4N, Narishige Co., Ltd.), the scalp was shaved and cut carefully with a surgical knife to expose the thin skull (calvaria) over the bilateral cerebral and cerebellar hemispheres without causing brain trauma. Using LSF (Omegazone; Omegawave, Inc.), baseline rCBF values were recorded for 20 seconds. The mouse was then placed supine, and ischemia was induced. The neck was incised at the midline between the manubrium and the jaw, and the left CCA was carefully separated from the vagus nerve. The external carotid artery (ECA) was ligated near its bifurcation into the lingual and maxillary arteries and cauterized. The occipital artery was cauterized and cut. The pterygopalatine artery was then exposed and isolated. A collar suture at the base of the ECA was prepared using a 5-0 silk suture, the internal carotid artery (ICA) was closed using a vascular clip, and the CCA was temporarily ligated with a 7-0 nylon suture. A silicone rubber-coated monofilament (coating length $4 \mathrm{~mm}$, tip diameter $0.21 \pm 0.02 \mathrm{~mm}$; Doccol Corporation) was introduced into the arteriotomy hole in the ECA and advanced into the left CCA bifurcation. After cutting the ECA, the suture was advanced distally into the ICA. Once the tip of the inserted suture reached the ICA, the collar suture of the ECA was tightened to avoid bleeding from the arteriotomy, and blood flow from the ICA was restored. The temporary occlusion time of the ICA was less than 1 minute. The intraluminal suture was inserted into the ICA $9 \pm 0.5 \mathrm{~mm}$ from the CCA bifurcation until mild resistance was felt. The collar suture at the base of the ECA stump was tightened securely around the inserted filament. The wound was closed using nylon sutures, with the intraluminal suture hidden in the wound, and the mouse was placed prone. rCBF values were recorded 3 minutes after ischemia using LSF for 20 seconds, and the ischemic duration was measured from the insertion of the silicone rubber-coated monofilament to the ICA. After a predetermined interval ranging from 5 to 45 minutes with the mouse in a supine position, the cervical wound was reopened and the suture was removed. After the ECA was ligated and blood flow of the CCA was reperfused, the wound was closed. The mouse 
was replaced in the prone position again, and rCBF values were recorded using LSF for 20 seconds. Each animal was then placed in a warm chamber $\left(33^{\circ} \mathrm{C}\right)$ for 24 hours after surgery and then returned to its cage at room temperature to ensure that its core temperature remained consistently at $37^{\circ} \mathrm{C} .{ }^{24}$

\section{Measurement of rCBF in the PCA Territory}

Real-time topographic rCBF images were obtained by LSF. rCBF was recorded through the intact skull. The periosteum, which adheres to the skull, was widely and gently removed using fine-tip forceps, and the temporal muscle was left intact. The surface of the skull was wiped with saline and covered with a slipcover over a thin layer of gel (Aquasonic; Parker Laboratories, Inc.) to prevent drying. During the rCBF measurements, the skull surface was illuminated diffusely by a $780-\mathrm{nm}$ laser light. Scattered light was filtered and detected using a charge-coupled-device camera positioned above the head. The filter detected only scattered light with perpendicular polarization to the incident laser light. Raw speckle images were used to calculate the speckle contrast, which corresponded to the number and velocity of moving red blood cells and was directly related to rCBF. Signal processing was performed by using an algorithm developed by Forrester et al. ${ }^{5}$ These experimental settings enabled us to measure the relative multipoint $\mathrm{rCBF}$ of the dorsal surface of the cerebrum (Fig. 1A). Color-coded blood-flow images were obtained in the high-resolution mode $(638 \times 480$ pixels; 1 image/ second). The mean $\mathrm{rCBF}$ was measured using regions of interest (ROIs) (7500 pixels) located in the PCA territory on the ischemic side (Fig. 1B and C). The absolute value of the $\mathrm{rCBF}$ was previously shown to be theoretically vulnerable to many experimental conditions, such as magnification of the charge-coupled-device camera, room light, and the amount of gel over the skull, which suggests that it was not always reliable. ${ }^{2}$ Therefore, we introduced the $\%$ rCBF of an ROI expressed as a percentage of the $\mathrm{rCBF}$ value of the ipsilateral ROI in the PCA territory against that of the corresponding ROI in the contralateral hemisphere. The PCA territory was defined, as previously reported, ${ }^{1}$ as that within $1.0 \mathrm{~mm}$ of the line parallel to the transverse sinus in the pseudo-color image obtained from LSF.

\section{Blood Gas Analysis}

In a separate group of mice, each subjected to 15 minutes of ischemia with a 4-mm silicone-coated suture (independent from widths and values of $\mathrm{rCBF}, \mathrm{n}=5$ ), arterial blood samples withdrawn through a PE-10 cannula inserted into the left femoral artery were analyzed before ischemia, 10 minutes after ischemia, and 10 minutes after reperfusion with a blood gas analyzer (i-STAT 1; FUSO Pharmaceutical Industries, Ltd.).

\section{Histological Evaluation of Neuronal Injury in the Hippocampal Medial CA1 Subregion}

Each mouse was anesthetized using $4 \%$ halothane exposure 1,4 , or 7 days after $5,10,15$, or 45 minutes of ischemia induced by a 4-mm silicone-coated intraluminal suture and transcardially perfused with buffered $10 \%$ for- malin. After postfixation for 24 hours, the brains were removed and embedded in paraffin. Coronal sections ( $4 \mu \mathrm{m}$ thick) containing the dorsal hippocampi were stained with $\mathrm{H} \& \mathrm{E}$, and the number of intact neurons in the hippocampal medial CA1 subregion (the region $1000 \mu \mathrm{m}$ from the end of the medial CA1 side) was counted in a blinded fashion. The average neuronal density calculated from the ischemic hemisphere was used to evaluate ischemic injury. Animals subjected to the same surgery without the insertion of a silicone-coated suture into the ICA served as controls.

\section{Evaluation of the Plasticity of the PCoA on the Ischemic Side and $\mathrm{rCBF}$ Images Using LSF}

rCBF in the PCA territory during experimental ischemia and reperfusion was measured to evaluate the correlation between PCoA plasticity and $\mathrm{rCBF}$ values of the PCA territory visualized by LSF during intraluminal arterial occlusion using a 4-mm coated length of suture. The mice were killed and intracardially perfused with $50 \mathrm{ml}$ of saline perfusion immediately after the rCBF measurement. A total of $0.8 \mathrm{ml}$ of carbon-black gelatin in an equal volume of $20 \%$ gelatin in $\mathrm{H}_{2} \mathrm{O}$ was then injected through the same intracardial cannula. To evaluate the patency of the PCoA, the brain was removed and the ipsilateral PCoA was analyzed microscopically.

\section{Neurological Scoring (Motor Activity)}

A 4-point neurological severity score was used to evaluate motor activity (1, no deficit; 2 , forelimb weakness; 3 , inability to bear weight on the affected side; and 4, no spontaneous motor activity). Mice in which the rCBF value of the PCA territory was less than $65 \%$ and those that received the sham surgery were evaluated neurologically 1 and 4 days after reperfusion.

\section{2,3,5-Triphenyltetrazolium Chloride Staining}

Brain infarcts 24 hours after 15 and 45 minutes of PCA ischemia were assessed by 2,3,5-triphenyltetrazolium chloride (TTC) staining as previously described with some modifications. ${ }^{1}$ Briefly, each mouse was decapitated after perfusion with saline. The brain was removed, and coronal sections $(2 \mathrm{~mm})$ were prepared. These sections were incubated for 20 minutes in $1 \%$ TTC in phosphatebuffered saline (PBS) at $37^{\circ} \mathrm{C}$ and then fixed with $4 \%$ paraformaldehyde for 24 hours. The brain slices were photographed using a Sony digital camera and evaluated using Photoshop CS6. Unstained areas were defined as infarcts.

\section{Terminal Deoxynucleotidyl Transferase-Mediated Deoxyuridine Triphosphate Nick-End Labeling Staining}

DNA fragmentation was detected by terminal deoxynucleotidyl transferase-mediated deoxyuridine triphosphate nick-end labeling (TUNEL) using a commercial kit (cata$\log$ 11684817910; Roche Diagnostics). As described above, paraffin sections were prepared 4 days after 5,10 , or 15 minutes of PCA ischemia. Sections were stained according to the manufacturer's protocol. Briefly, the sections were deparaffinized and treated by ethylenediaminetet- 

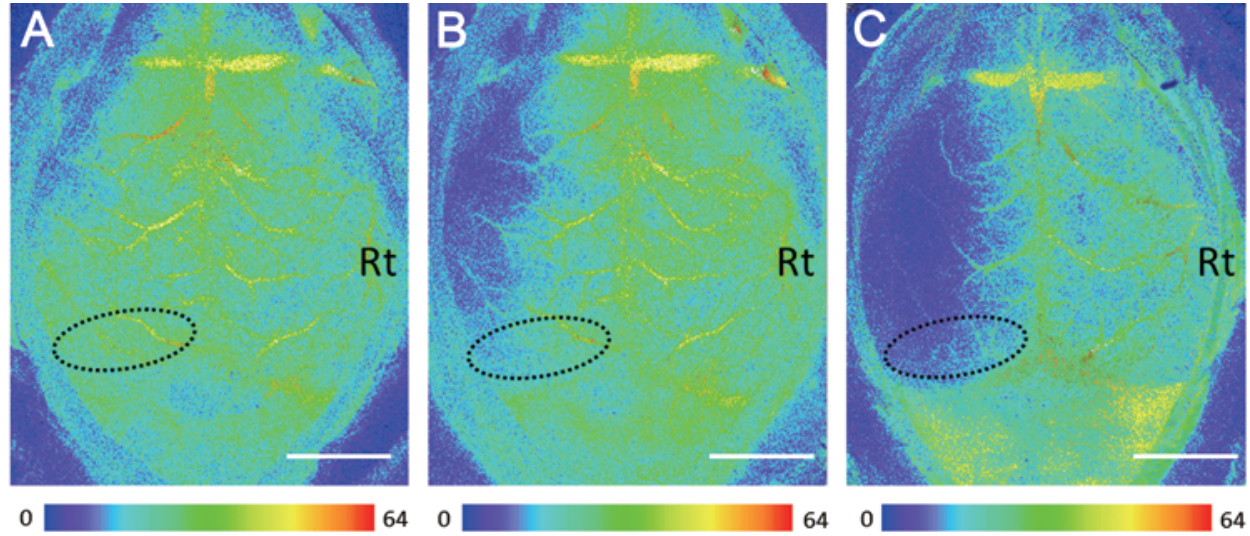

0
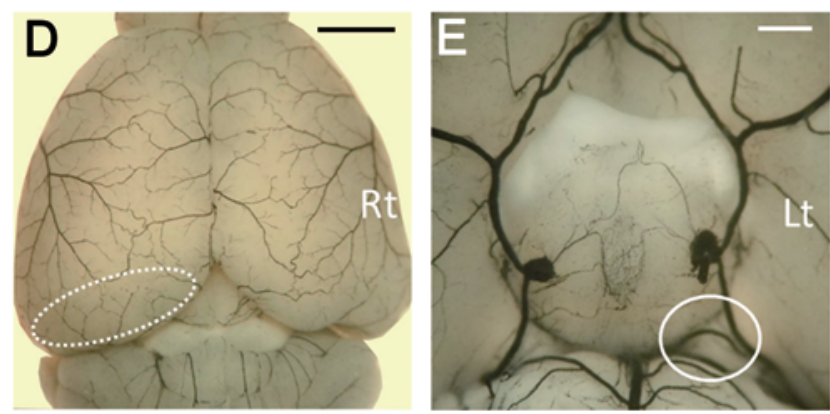

64

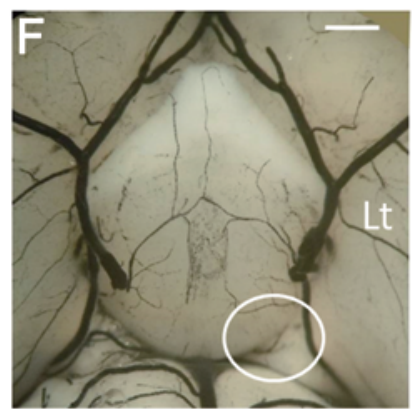

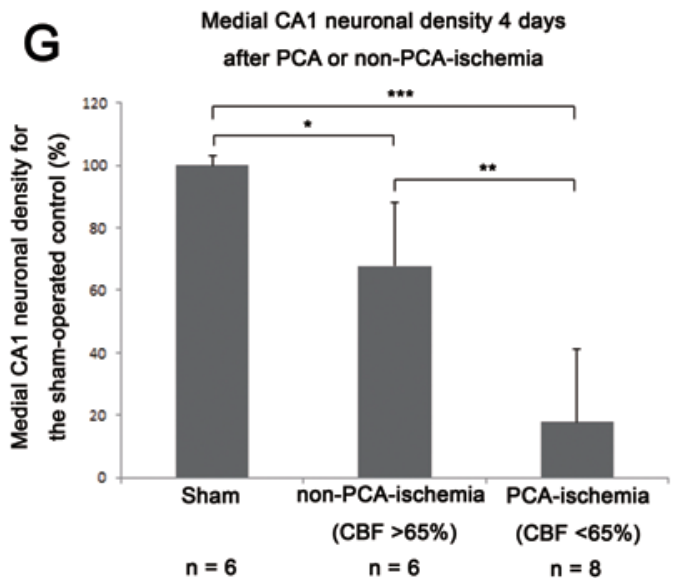

FIG. 1. A-F: Photographs showing the topographic rCBF images using the 64-level color scale obtained from LSF (A-C) and the brain surface and PCoA plasticity visualized with carbon-black gelatin (D-F). Rt = Right, Lt = left. A and D: Images obtained from a mouse that did not have surgery. The PCA territory is surrounded by dotted circles. B and E: Photographs obtained from a mouse with developed PCoA on the ischemic side. E: A well-developed PCoA was made visible by carbon-black dye (circle). B: The corresponding rCBF image shows that rCBF in the PCA territory (dotted circle) was preserved after insertion of the intraluminal suture $(\mathrm{rCBF}>65 \%)$. In contrast, photographs obtained from a mouse with a hypoplastic PCoA (F, circle) on the ischemic side ( $C$ and $F$ ) show that $r C B F$ in the PCA territory was reduced significantly after insertion of the intraluminal suture $(r C B F<65 \%)$. Bars $=3 \mathrm{~mm}(\mathbf{A}-\mathbf{D})$ and $1 \mathrm{~mm}(\mathbf{E}$ and $\mathrm{F})$. G: Quantitative evaluation of medial CA1 neuronal injury in C57BL/6 mice. The CA1 neuronal count was compared between the controls that received sham surgery $(n=6)$ and groups showing $\mathrm{rCBF}$ values of $>65 \%$ $(n=6)$ and $<65 \%(n=8)$ in the PCA territory 4 days after ischemia. The number of surviving neurons was significantly lower in the group with $\mathrm{rCBF}$ values of $<65 \%$ than in the controls that received sham surgery. All values are expressed as mean \pm SD. ${ }^{*} p<$ 0.05 vs sham; ${ }^{* *} p<0.001$ vs the group with rCBF values of $>65 \% ;{ }^{* * *} p<0.0001$ vs sham. Figure is available in color online only.

raacetic acid solution while being heated in a microwave. Then, the sections were reacted in a solution of terminal deoxynucleotidyl transferase enzyme mixed with biotinylated nucleotide. Horseradish peroxidase-labeled streptavidin was then bound to these biotinylated nucleotides, which were detected using the peroxidase substrate, hydrogen peroxide, and diaminobenzidine (DAB). All the sec- tions were counterstained with hematoxylin. To quantify TUNEL staining in the CA1 subregion, TUNEL-positive cells were counted in a $1000-\mu \mathrm{m}$ length of the medial portion of the CA1 subregion on the ischemic side, and the percentage of TUNEL-positive cells for intact neurons in the medial CA1 subregion of controls that received sham surgery were calculated. 


\section{Cleaved Caspase 3 Immunohistochemistry}

Paraffin sections from mice 4 days after 15 minutes of ischemia and from those that received the sham surgery were prepared as described above and labeled with cleaved caspase 3 . In brief, sections were deparaffinized and treated with ethylenediaminetetraacetic acid solution combined with heating in a microwave. Sections were then washed in $0.01 \mathrm{M}$ PBS and quenched for 10 minutes in a solution of methanol containing 3\% hydrogen peroxide. Nonspecific binding was blocked with blocking solution (2\% bovine serum albumin, $0.2 \%$ milk, and $0.1 \%$ Triton X-100 in PBS) for 1 hour. Sections were immunostained with a 1:300 dilution of an anti-activated caspase 3 (Asp-175) antibody (catalog no. 9661; Cell Signaling Technology) followed by biotin-conjugated goat anti-rabbit immunoglobulin $\mathrm{G}$ at a dilution of 1:200 in blocking solution. They were then reacted in the dark with ABC (avidin-biotin complex) reagents (standard Vectastain $\mathrm{ABC}$ Elite kit; Vector Laboratories, Inc.) and preincubated for $10 \mathrm{~min}$ in a filtered mixture containing $6 \mathrm{ml}$ of $0.1 \mathrm{M}$ Tris buffer, $2 \mathrm{mg} \mathrm{DAB}$, and $400 \mathrm{mg}$ imidazole and then for 15 minutes in $6 \mathrm{ml}$ of the same DAB-imidazole-Tris mixture containing $3 \mathrm{ml}$ of $\mathrm{H}_{2} \mathrm{O}$. All sections were counterstained with hematoxylin.

\section{Statistical Analysis}

All values are expressed as means \pm the standard deviation (SD). Comparisons among multiple groups, except those for neurological scores, were performed with an analysis of variance followed by Bonferroni post hoc analysis. Comparisons of the neurological scores were performed with the Kruskal-Wallis test with the Dunn post hoc test. Comparisons between 2 groups were achieved with an unpaired Student t-test. $p$ values of $<0.05$ were considered significant. All statistical analyses were performed on a personal computer using GraphPad Prism for Microsoft Windows version 6 (GraphPad Software, Inc.).

\section{Results}

\section{Correlation Between rCBF Values on the PCA Territory, PCoA Plasticity, and CA1 Neuronal Death}

Topographic rCBF images obtained from LSF allowed us to monitor in real time whether the current ischemic insult affected the PCA territory (Fig. 1A-C). Topographic CBF images on the PCA territory obtained from LSF were correlated with PCoA plasticity, made visible with carbonblack gelatin (Fig. 1D-G). Mice with high rCBF values (> $65 \%$ ) in the PCA territory had a developed PCoA (Fig. 1B and $\mathrm{E})$. In contrast, mice with low rCBF values $(<65 \%)$ in the PCA territory showed poor patency of the PCoA (Fig. 1C and F). Therefore, we established the cutoff rCBF value as $65 \%$ and defined mice that had an $\mathrm{rCBF}$ value of less than $65 \%$ in the PCA territory as PCA-ischemic mice. We used PCA-ischemic mice in the following study and histologically evaluated them at different time points after ischemia. Medial CA1 neurons were more injured 4 days after 15 minutes of ischemia in PCA-ischemic mice (rCBF $<65 \%)$ than in non-PCA-ischemic mice $(\mathrm{rCBF}>65 \%)$ (Fig. 1G). On the basis of these results, we considered the possibility that an $\mathrm{rCBF}$ value of $<65 \%$ in the PCA terri- tory (evaluated by the LSF) may induce hippocampal CA1 neuronal death 4 days after ischemia in C57BL/6 mice. Thus, we subsequently performed histological evaluations at different time courses and with different ischemic durations using PCA-ischemic mice.

\section{Neuronal Death in the Medial CA1 Subregion After 15 Minutes of PCA Ischemia}

We next investigated CA1 neuronal death after 15 minutes of PCA ischemia with time-course samples. Consistent medial CA1 neuronal death was observed 4 days after PCA ischemia. Neuronal injury in the medial CA1 subregion peaked 4 days after 15 minutes of PCA ischemia (Fig. 2A and B). Most pyramidal neurons exhibited pyknotic and

A
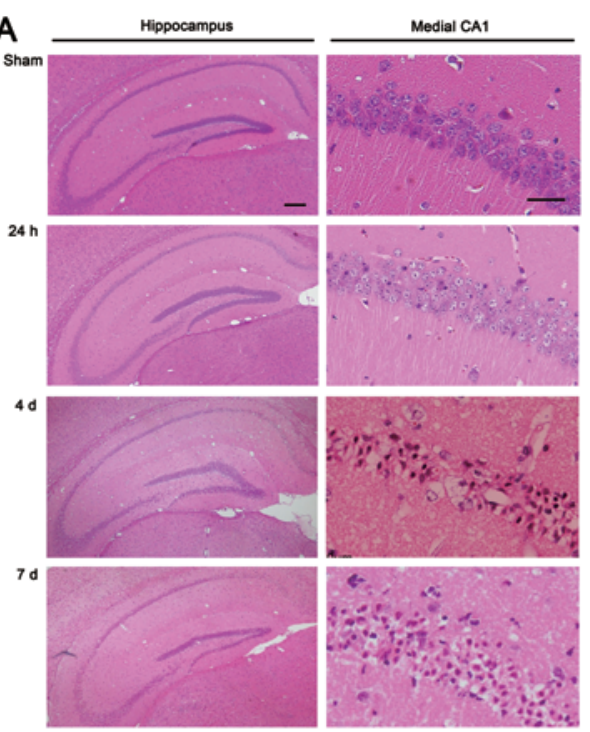

B
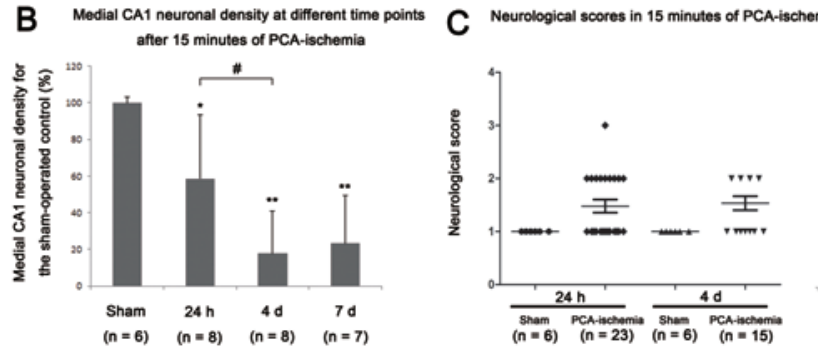

FIG. 2. A: Representative photomicrographs of $H$ \& $E$ staining in the hippocampus and medial CA1 subregion at each time point after 15 minutes of PCA ischemia. Medial CA1 neurons were uniformly shrunken 4 and 7 days after ischemia but not 1 day after ischemia, and there were slight morphological changes relative to those in controls that received sham surgery. Bars $=200 \mu \mathrm{m}$ (left column) and $50 \mu \mathrm{m}$ (right column). B: Medial CA1 neuronal counts after 15 minutes of PCA ischemia were evaluated at different time points after ischemia ( 24 hours $[n=8], 4$ days $[n=8]$, and 7 days [ $n=7]$ ), and neuronal densities were calculated for controls that received sham surgery $(n=6)$. Severe and significant reductions in neuronal density were observed 4 and 7 days after ischemia. All values are expressed as mean \pm SD. ${ }^{*} p<0.05$ vs sham; ${ }^{* *} p<0.0001$ vs sham; $\# p<0.05$ vs 24 hours. C: Neurological deficits assessed 24 hours $(n=23)$ and 4 days $(n=15)$ after PCA ischemia by the 4-point neurological severity scale. No significant differences were observed between the groups 24 hours and 4 days after ischemia and the controls that received sham surgery $(n=6) . h=$ hours; $d=$ days. Figure is available in color online only. 
shrunken nuclei 4 and 7 days after 15 minutes of PCA ischemia. In contrast, approximately half of the neurons were not degenerated 24 hours after PCA ischemia.

We also evaluated neurological scores after PCA ischemia at each time point. Neurological scores tended to be high after PCA ischemia but were not significantly different in the groups 24 hours and 4 days after PCA ischemia compared with those of the controls that received sham surgery (Fig. 2C).
In summary, in this time-course study, delayed medial CA1 neuronal death, such as delayed neuronal death after tGCI, was observed after 15 minutes of PCA ischemia in C57BL/6 mice.

\section{Severity of Neuronal Death in the Medial CA1 Subregion Increased With Ischemic Duration}

We demonstrated delayed unilateral hippocampal neuronal death after at least 15 minutes of PCA ischemia. We
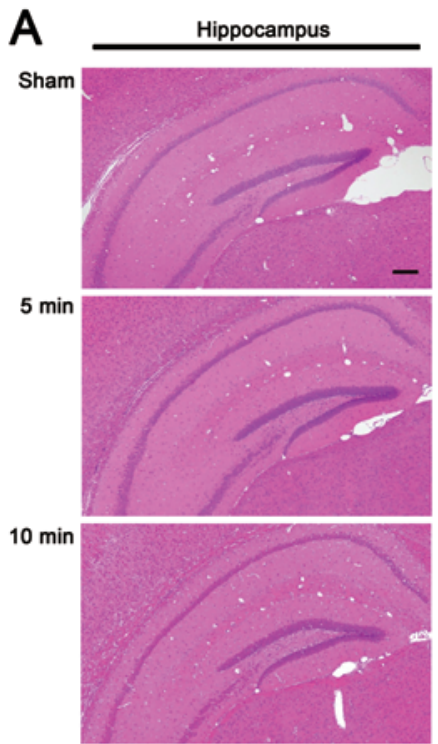

$15 \mathrm{~min}$

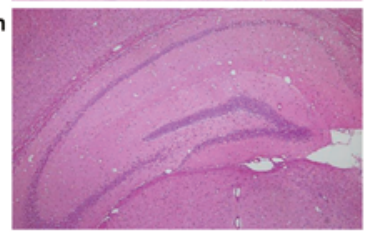

B

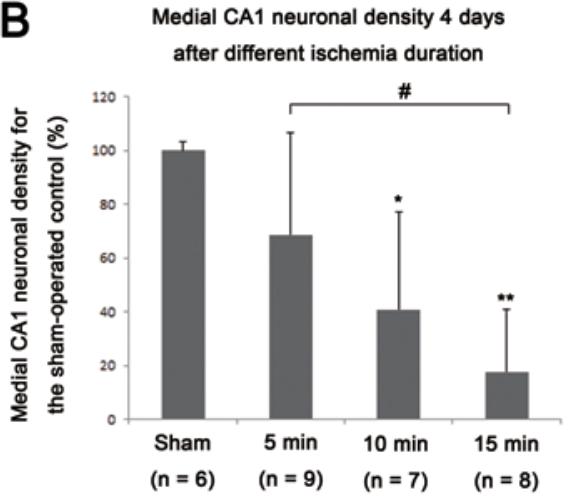

Medial CA1
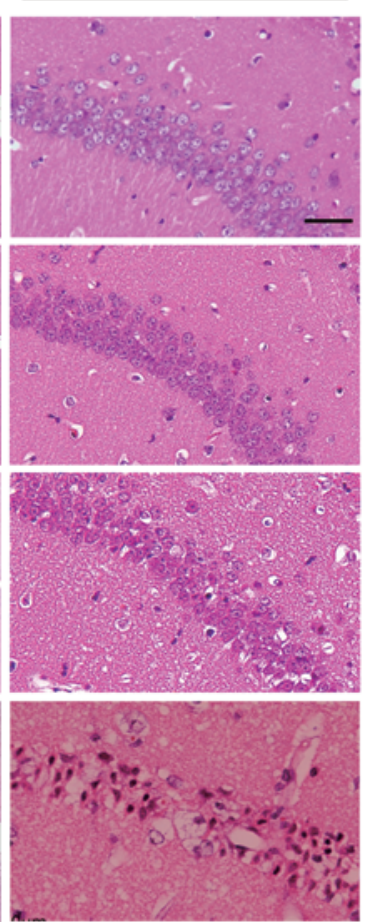
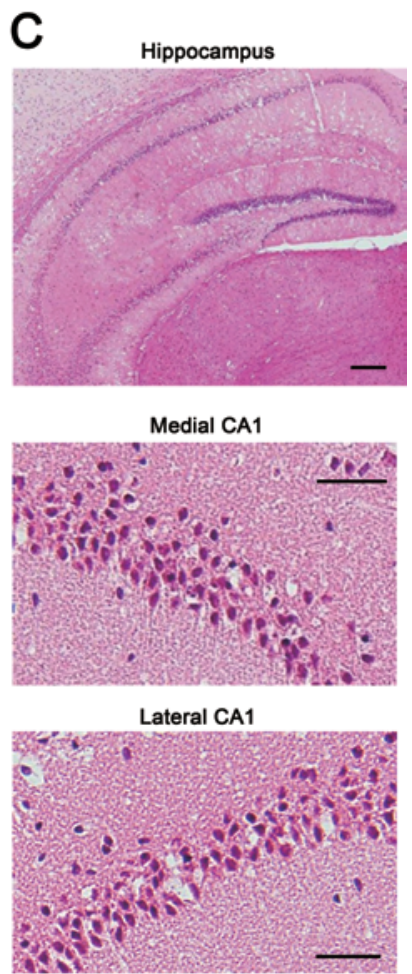

D
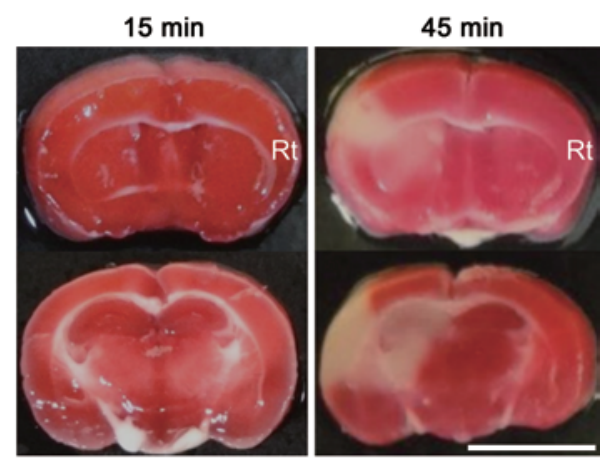

FIG. 3. A: Representative photomicrographs of $\mathrm{H} \& \mathrm{E}$ staining in the hippocampus and medial $\mathrm{CA} 1$ subregion 4 days after different durations of PCA ischemia. Medial CA1 neurons were uniformly shrunken after 15 minutes of ischemia. Bars $=200 \mu \mathrm{m}$ (left column) and 50 um (right column). B: The medial CA1 neuronal count was compared between controls that received sham surgery and the groups subjected to 5-15 minutes of PCA ischemia 4 days after ischemia. The numbers of surviving neurons were not significantly less than those of controls that received sham surgery $(n=6)$ after 5 minutes $(n=9)$ of ischemia but were less after $10(n=7)$ and $15(n=8)$ minutes of ischemia. All values are expressed as mean $\pm S D$. ${ }^{*} p<0.01$ versus sham; ${ }^{* *} p<0.001$ versus sham; $\# p<0.05,5$ minutes versus 15 minutes. C: Representative photomicrographs of $H$ \& $E$ staining in the hippocampus after 24 hours in the group that experienced 45 minutes of PCA ischemia $(n=4)$. Necrotic cells and marked brain swelling were observed in the whole hippocampal region. Bars $=200 \mu \mathrm{m}$ (upper) and $50 \mu \mathrm{m}$ (center and lower). D: TTC-stained coronal sections 24 hours after PCA ischemia. No infarctions were found in any brain areas after 15 minutes of ischemia. In contrast, pannecrosis was observed after 45 minutes of ischemia. Bar $=5 \mathrm{~mm}$. Figure is available in color online only. 
then investigated the role of ischemic duration in inducing hippocampal neuronal death. Medial CA1 neuronal injury 4 days after ischemia increased as the ischemic duration increased (Fig. 3A and B). Most pyramidal neurons exhibited pyknotic shrunken nuclei 4 days after 15 minutes of PCA ischemia, and the densities of medial CA1 neurons were significantly less than those after 5 and 10 minutes of PCA ischemia (Fig. 3A). Necroses, which were observed after typical MCAO, were not observed after 5-15 minutes of PCA ischemia. In contrast, pan-necroses were observed in the hippocampus and other MCA territories 24 hours after PCA ischemia in all mice in the group that experienced 45 minutes of PCA ischemia (Fig. 3C). In addition, no infarctions were observed in TTC-stained brains after 15 minutes of PCA ischemia, but pan-necroses were observed after 45 minutes of PCA ischemia (Fig. 3D). These results indicate that the longer PCA-ischemia duration induced slightly more severe delayed neuronal cell death in the medial CA1 subregion. However, longer ischemia durations may cause hippocampal infarction. Fifteen minutes of PCA ischemia produces a reasonable level of neuronal cell death in the medial CA1 subregion.

\section{TUNEL Staining and Cleaved Caspase 3 Immunohistochemistry}

To evaluate the mechanism of this delayed unilateral CA1 neuronal death, TUNEL staining and immunohistochemistry of cleaved caspase 3 were performed 4 days after 15 minutes of PCA ischemia. TUNEL- and cleaved caspase 3-positive cells were observed in the hippocampal CA1 subregion 4 days after 15 minutes of PCA ischemia (Fig. 4A). DNA fragmentation in mice 4 days after ischemia increased according to the ischemic duration (5 minutes, $13.5 \%$; 10 minutes, $22.2 \%$ ) and was significantly higher after 15 minutes of PCA ischemia than in mice after sham surgery $(48.6 \%$; $p<0.01)$ (Fig. 4B). These results indicate that apoptosis had contributed, at least in part, to delayed unilateral neuronal death in the medial CA1 subregion after 15 minutes of unilateral PCA ischemia.

Conversely, ischemic injuries were also observed in other regions of the hippocampus on the ischemic side 4 days after 15 minutes of PCA ischemia. TUNEL-positive cells were observed in the CA2-3 corner, the medial part of CA3, the hilus, and the dentate gyrus after 15 minutes of PCA ischemia (Fig. 5A-C). However, neither the severity nor the intensity of these injuries was consistent. Similarly, extrahippocampal ischemic injury was observed on the ischemic side. TUNEL-positive cells were also noted, mainly in layers 3-5 of the cortex, thalamus, and striatum, after 15 minutes of PCA ischemia (Fig. 5D-F). These ischemic changes in other lesions were similar to those in mice that experienced conventional tGCI. ${ }^{12,19,24,26,27}$

\section{Survival Rates and Reproducibility}

In this study, 74 mice underwent surgical procedures. Sixty-seven mice $(90.5 \%)$ survived and were subjected to $\mathrm{CBF}$ analysis. Among these 67 mice, $65.7 \%$ (44 of 67 procedures) demonstrated PCA ischemia $(\mathrm{rCBF}<65 \%$ in the PCA territory), and $34.3 \%$ (23 of 67) had non-PCA ischemia ( $\mathrm{rCBF}>65 \%$ in the PCA territory). The survival rates of mice subjected to 15 minutes of ischemia in the PCA-
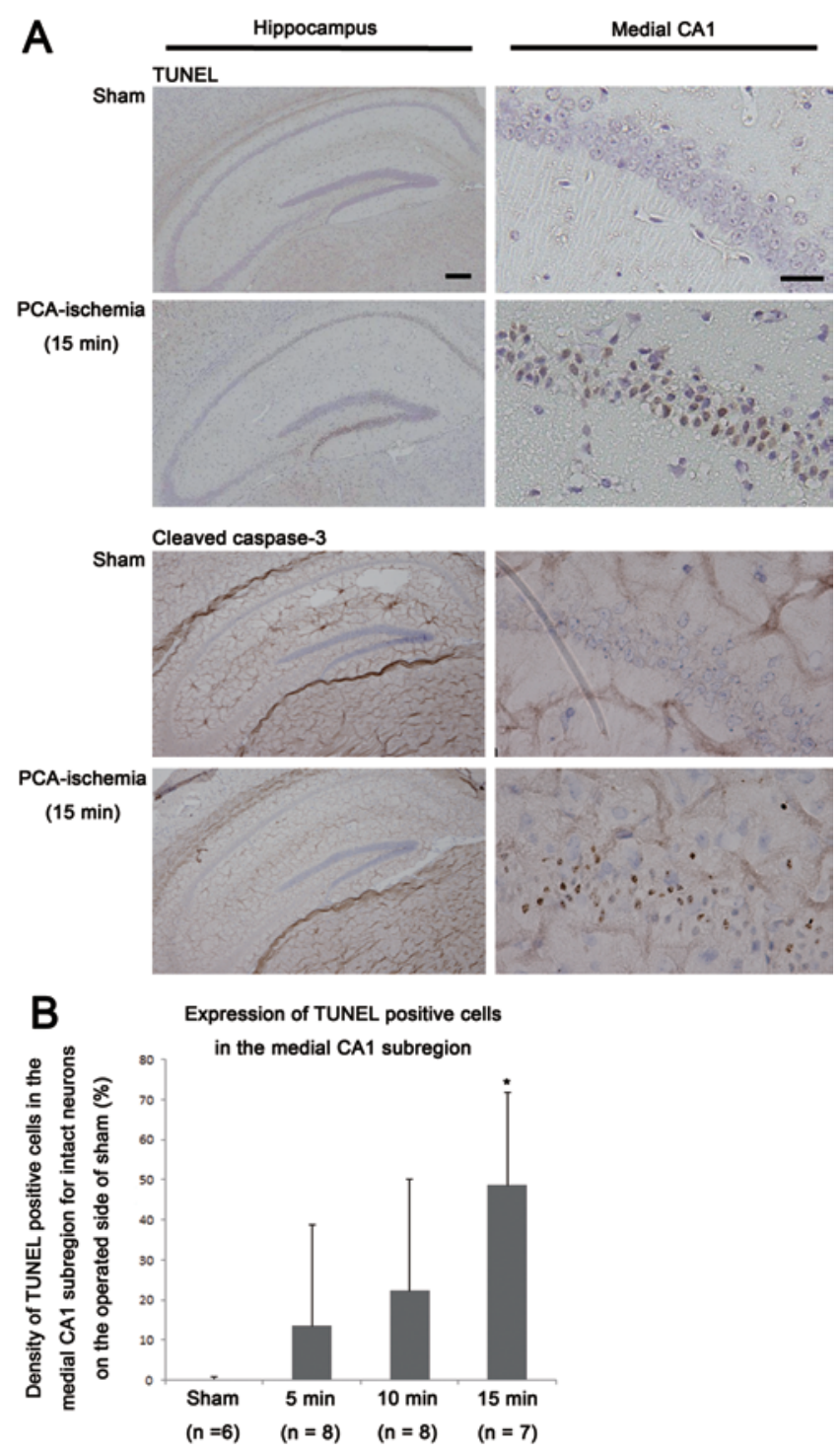

FIG. 4. A: Representative photomicrographs of TUNEL staining and immunohistochemistry of cleaved caspase 3 in the hippocampus and medial CA1 subregion 4 days after 15 minutes of PCA ischemia. TUNELand cleaved caspase 3-positive cells with shrunken nuclei were observed in the medial CA1 subregion. Bars $=200 \mu \mathrm{m}$ (left column) and 50 um (right column). B: Quantitative evaluation of TUNEL-positive cells in the medial CA1 subregion 4 days after different PCA-ischemia durations. A significant increase in the density of TUNEL-positive cells over that of the controls that received sham surgery $(n=6)$ was not observed after $5(n=8)$ or $10(n=8)$ minutes of ischemia but was observed after 15 minutes of ischemia $(n=7)$. All values are expressed as mean \pm SD. ${ }^{* *} p<0.01$ vs sham. Figure is available in color online only.

ischemic group were $95.8 \%$ at 24 hours $(n=23$ of $24[1$ mouse died as a result of hemorrhagic infarction]), $100 \%$ at 4 days $(\mathrm{n}=15)$, and $100 \%$ at 7 days $(\mathrm{n}=7)$. Consistent medial CA1 neuronal death was observed 4 and 7 days after PCA ischemia (Fig. 2B).

\section{Physiological Parameters}

Physiological parameters were not significantly different between PCA-ischemic ( $\mathrm{rCBF}<65 \%$ in the PCA ter- 

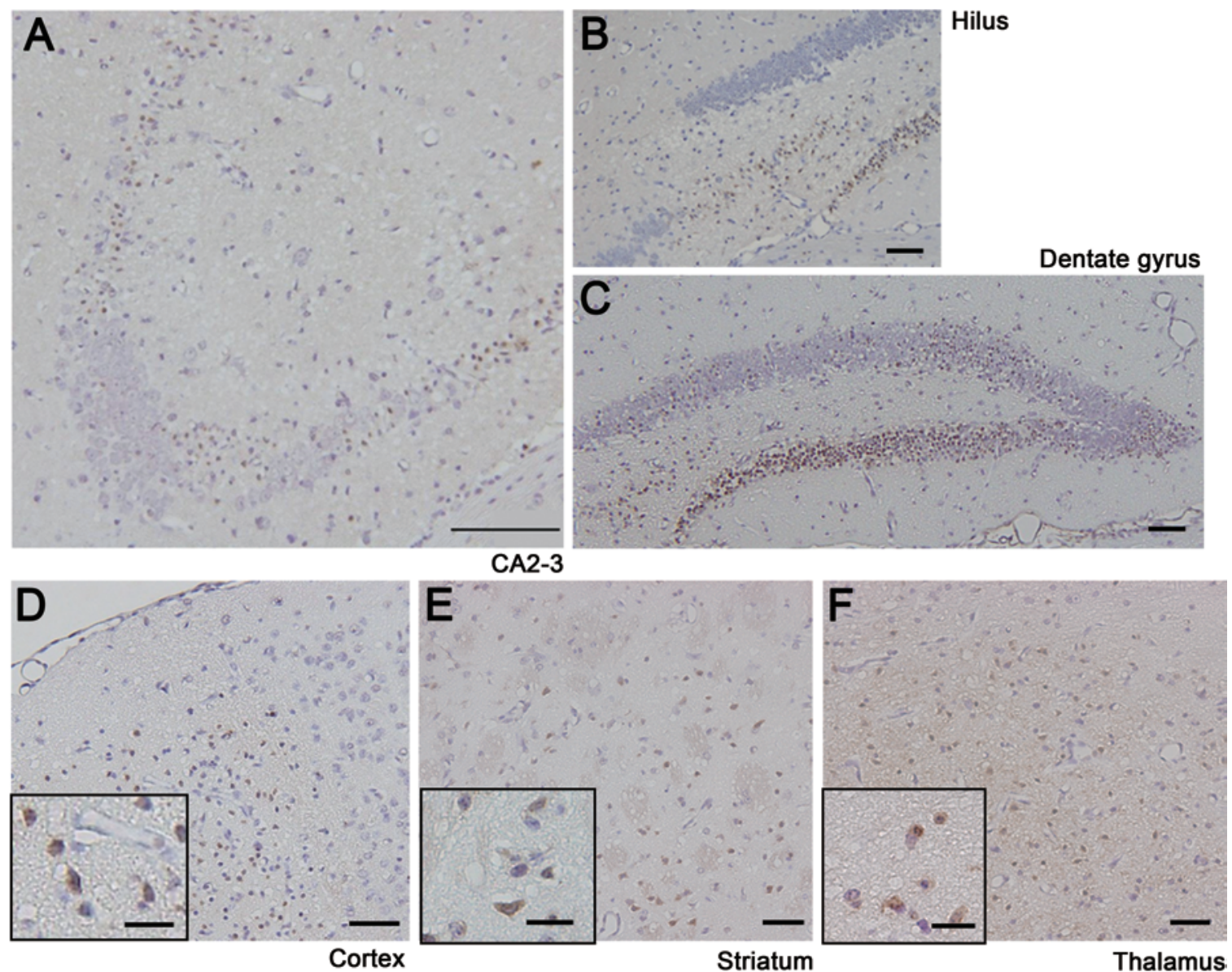

FIG. 5. Ischemic injuries in other than CA1 subregions of the hippocampus and extrahippocampal regions on the ischemic side. A-C: TUNEL-positive cells were observed in the CA2-3 corner and the medial part of CA3 (A), the hilus (B), and the dentate gyrus (C) 4 days after 15 minutes of PCA ischemia. D-F: TUNEL-positive cells were observed outside of the hippocampus in the cortex (D), striatum (E), and thalamus (F) after 4 days. Bars $=100 \mu \mathrm{m}(A-C), 50 \mu \mathrm{m}(D-F)$, and $20 \mu \mathrm{m}$ (insets in D-F). Figure is available in color online only.

ritory) and non-PCA-ischemic (rCBF $>65 \%$ in the PCA territory) mice and the controls that received sham surgery during the procedure in this model, except for the $\mathrm{rCBF}$ values at the time of ischemia (Table 1). Blood gas data measured in the separate groups 15 minutes after ischemia are shown in Table 2. The $\mathrm{pH}$ level was lower and the $\mathrm{CO}_{2}$ level was higher after reperfusion than those before or during ischemia.

\section{Discussion}

In this study, we used a modified MCAO model that did not cause infarction of the MCA territory but did result in hippocampal ischemia and induced consistent neuronal death at the unilateral medial CA1 subregion in C57BL/6 mice. This model was established by using a simple intraluminal suture method widely used for MCAO models in mice, monitoring the topographic PCA-territory $\mathrm{CBF}$ values using LSF, and selecting mice with PCA ischemia (mice with a hypoplastic PCoA) that indirectly reflected noncollateral flow to the hippocampus during PCA occlusion. TTC staining revealed no acute infarction in any brain areas 24 hours after 15 minutes of PCA ischemia.
On the other hand, TUNEL and cleaved caspase 3 staining indicated that apoptosis played a role, at least in part, in unilateral delayed neuronal death in the hippocampal CA1 subregion after unilateral PCA ischemia. This model also affected territories including the CA2-3 corner, the dentate gyrus, the cerebral cortex of the MCA territory, the striatum, and the thalamus. These histological changes resembled conventional global cerebral ischemia in mice. ${ }^{13,20,25,27,28}$

Many researchers have attempted to produce a mouse model of global cerebral ischemia to ensure consistent CA1 injury. The simplest and most widely used model is the bilateral CCA occlusion (BCCAO) model. However, the disadvantage of this model is the inconsistent degrees of ischemic injury that result from individual variance in collateral blood flow through the circle of Willis. The irregular occurrence of the PCoA was observed even in the

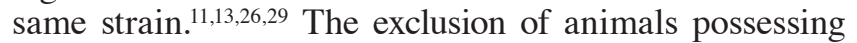
the PCoA was advantageous in increasing the reproducibility of CA1 neuronal death by the BCCAO method. .11,13,28,29 $^{1}$ However, difficulties remain in attaining uniform injury to the $\mathrm{CA} 1$ region while retaining high survival and success rates. 
TABLE 1. Physiological parameters*

\begin{tabular}{|c|c|c|c|c|}
\hline Parameter & Group & Preischemia & During Ischemia & Postischemia \\
\hline \multirow[t]{3}{*}{$\operatorname{MABP}(\mathrm{mm} \mathrm{Hg})$} & Sham surgery† & $62.5 \pm 3.9$ & $68.3 \pm 8.2$ & $63.3 \pm 6.3$ \\
\hline & Non-PCA ischemia† & $62.8 \pm 5.2$ & $61 \pm 4.5$ & $59.1 \pm 2.0$ \\
\hline & PCA ischemia† & $64.1 \pm 3.5$ & $65.1 \pm 6.2$ & $60.75 \pm 4.6$ \\
\hline \multirow[t]{3}{*}{$\mathrm{RT}\left({ }^{\circ} \mathrm{C}\right)$} & Sham surgery† & $36.6 \pm 0.1$ & $36.7 \pm 0.1$ & $36.7 \pm 0.1$ \\
\hline & Non-PCA ischemia† & $36.6 \pm 0.1$ & $36.7 \pm 0.1$ & $36.7 \pm 0.1$ \\
\hline & PCA ischemia† & $36.7 \pm 0.2$ & $36.8 \pm 0.2$ & $36.8 \pm 0.2$ \\
\hline \multirow[t]{3}{*}{ rCBF (\%) } & Sham surgery† & $105.9 \pm 5.1$ & $96.3 \pm 6.4$ & $100.8 \pm 2.9$ \\
\hline & Non-PCA ischemia† & $100.9 \pm 2.7$ & $81.0 \pm 11.1 \ddagger$ & $100.5 \pm 7.8$ \\
\hline & PCA ischemia† & $103.2 \pm 4.7$ & $59.8 \pm 3.9 \ddagger$ & $98.24 \pm 9.9$ \\
\hline $\begin{array}{l}\text { MABP }=\text { mean arterial blc } \\
{ }^{*} \text { All values are expresse } \\
\text { † Sham-operated group } \\
(n=6) .\end{array}$ & $\begin{array}{l}\text { Ire; } R T \text { = rectal temperat } \\
\text { Is } \pm \text { SD. } \\
\text { ion-PCA-ischemia: rCB }\end{array}$ & $\begin{array}{l}\text { he PCA territ } \\
\text {.01). }\end{array}$ & $\mathrm{CA}$ ischemia: $\mathrm{rC}$ & in the PCA territory \\
\hline
\end{tabular}

Another approach that has been used is to decrease $\mathrm{CBF}$ by a combination of $\mathrm{BCCAO}$ and a reduction in blood flow via vertebrobasilar arteries. This approach was used in a 3-vessel occlusion model in which the occlusion of carotid arteries was combined with clamping of the basilar artery. ${ }^{16,27}$ However, the animal survival rate was not as high as previously reported, and CA1 neuronal injury was inconsistent. ${ }^{16}$ Although Yonekura and colleagues ${ }^{27}$ achieved consistent uniform neuronal injury in the CA1 region in C57BL/6 mice with modified 3-vessel occlusion, the technical difficulties associated with this method have limited its application.

An alternative method is BCCAO with hypotension to minimize collateral blood flow by the withdrawal of blood via a venous catheter inserted into the jugular vein or by the induction of a high dose of isoflurane..$^{15,20,21,25}$ However, among these models, that of BCCAO with hypotension induced by the withdrawal of blood required a more complex process for cannulation of both the femoral (or tail) artery and jugular vein. It may lead to hind limb ischemia on the side ipsilateral to the arterial catheter ${ }^{25}$ and influence behavioral studies. In addition, systemic hypotension may damage other organs such as the kidney and heart, which may play a role in physiological mechanisms of postischemic survival. $^{18}$

In previous studies of cardiac arrest in mice, Kawahara and colleagues ${ }^{8}$ developed a 5-minute cardiac arrest model in C57BL/6 mice, which was not affected by the vascular anatomy, and evaluated hippocampal neuronal injury. However, no consistent ischemic neuronal injury was noted in the CA1 region of the hippocampus with this 5-minute cardiac arrest. They then extended circulatory arrest to 6 minutes; however, the mortality rate reached more than $90 \%$, which highlighted a limitation of their cardiac arrest model in mice. They suggested that murine hippocampal neurons were more resistant to ischemia than those in other rodents. This result showed that a longer ischemic duration is needed to induce neuronal injury in the hippocampal CA1 subregion of mice than that of other rodents.

Considering these previous studies, the major problems associated with a mouse model of delayed hippocampal neuronal death were inconsistent degrees of hippocampal injury and high mortality rates. These were based on differences in collateral blood flow through the circle of Willis, technical difficulties of the models, and/or resistance of murine neurons to ischemia. Differences in collateral blood flow were normalized in our model by a simple method: excluding mice that possessed a developed PCoA, as determined by using LSF. The rCBF value of the cortical PCA territory measured by LSF was correlated with the plasticity of the PCoA. Because the ECA and MCA do not perfuse the hippocampus, the cortical rCBF value of the PCA territory measured by LSF reflects collateral blood flow from the PCoA during PCA ischemia caused by an intraluminal nylon suture.

Unilateral ischemic insult and the simple suture method of our model contributed to increased survival rates. Our success rate for surgery was $90.5 \%$. The survival rate of the 15-minute PCA-ischemic mice that showed CA1 neuronal death was $95.8 \%$, and all mice that survived 1 day after PCA ischemia survived 7 days after PCA ischemia (longer survival times were not examined). In contrast, the survival rates in previous BCCAO models were low $(75 \%$ after a 15-minute BCCAO in CD1 mice ${ }^{29}$ and $81.6 \%$ after a 22-minute $\mathrm{BCCAO}$ in $\mathrm{C} 57 \mathrm{BL} / 6$ mice (however, more than $50 \%$ of the surviving mice suffered from severe akinesia $\left.{ }^{28}\right)$. Even with the strict criteria for $\mathrm{rCBF}$, only $\leq 50 \%$ of the mice were found to have bilateral CA1 injury. ${ }^{11,28,29}$

In terms of neurological findings, the mice in our mod-

TABLE 2. Blood gas analysis*

\begin{tabular}{lccc}
\hline \multicolumn{1}{c}{ Parameter } & $\begin{array}{c}\text { Preischemia } \\
(n=5)\end{array}$ & $\begin{array}{c}\text { During Ischemia } \\
(n=5)\end{array}$ & $\begin{array}{c}\text { Postischemia } \\
(n=5)\end{array}$ \\
\hline$p H$ & $7.24 \pm 0.02$ & $7.23 \pm 0.02$ & $7.14 \pm 0.04 \dagger \ddagger$ \\
\hline $\mathrm{PaCO}_{2}(\mathrm{~mm} \mathrm{Hg})$ & $56.2 \pm 5.3$ & $57.5 \pm 4.2$ & $66.1 \pm 5.4 \dagger \S$ \\
\hline $\mathrm{PaO}_{2}(\mathrm{~mm} \mathrm{Hg})$ & $61.6 \pm 9.0$ & $58.2 \pm 9.9$ & $56.8 \pm 12.4$ \\
\hline
\end{tabular}

* All values are expressed as the mean \pm SD.

$\dagger$ Significantly different from the preischemia group $(p<0.01)$.

$\ddagger$ Significantly different from the ischemia group $(p<0.01)$.

$\S$ Significantly different from the ischemia group $(p<0.05)$. 
el had slight motor deficits. Global cerebral ischemia is known to produce impairments of motor behavior, mainly because of a severe reduction of striatal neurons. ${ }^{3}$ In our model, ischemic injuries were also observed in the striatum, which might cause deficits to show in neurological examinations.

We consider the advantages of our model to be the consistent degrees of hippocampal CA1 injury, the low mortality rate, and internal controls (contralateral nonischemic hippocampus). In addition, because of the low mortality rate with only slight motor deficits, this model may be used as a model for long-term survival of hippocampal ischemia to study not only the mechanism of delayed hippocampal neuronal injury but also neuronal regeneration, higher brain functions (such as memory, learning, and behavior), and the origins of epilepsy. Furthermore, we emphasize that internal controls in our model can always be created under the same conditions, which is not possible in the conventional tGCI model. The limitations of our study are as follows. First, although we tested motor activities by a 4-point neurological severity score, other behavioral assessments, such as of learning or memory, were not conducted. Second, the physiological parameters (blood gases) of each animal were not maintained at a more physiological level, because the ketamine-xylazine mixture was injected intraperitoneally for anesthesia. Third, brain temperature was not monitored or controlled, because skull exposure was needed for the measurement of CBF.

\section{Conclusions}

In this study, we established a model of delayed unilateral hippocampal neuronal death in C57BL/6 mice using a modified intraluminal suture method and included criteria for PCA-territory rCBF values monitored by LSF. This model consistently resulted in quantitatively uniform delayed unilateral neuronal death in the medial CA1 subregion and low mortality rates without severe akinesia, which allowed for long-term experiments. This model can provide internal controls, which contribute to analyzing the more precise mechanisms of ischemic neuronal injury. This model may be used to investigate the mechanisms of ischemic injury and to analyze neuronal regeneration or higher brain dysfunction, including cognitive deficits such as learning or memory disorders.

\section{Acknowledgments}

We thank Ms. Misaki Fue and Mr. Tomizo Shiratori for their technical assistance.

\section{References}

1. Akamatsu Y, Shimizu H, Saito A, Fujimura M, Tominaga T: Consistent focal cerebral ischemia without posterior cerebral artery occlusion and its real-time monitoring in an intraluminal suture model in mice. J Neurosurg 116:657-664, 2012

2. Ayata C, Dunn AK, Gursoy-Özdemir Y, Huang Z, Boas DA, Moskowitz MA: Laser speckle flowmetry for the study of cerebrovascular physiology in normal and ischemic mouse cortex. J Cereb Blood Flow Metab 24:744-755, 2004

3. de Araujo FL, Bertolino G, Gonçalves RB, de Castro Marini L, Coimbra NC, de Araujo JE: Neuropathology and be- havioral impairments after three types of global ischemia surgery in Meriones unguiculatus: evidence in motor cortex, hippocampal CA1 region and the neostriatum. J Neurol Sci 312:73-78, 2012

4. Dorr A, Sled JG, Kabani N: Three-dimensional cerebral vasculature of the CBA mouse brain: a magnetic resonance imaging and micro computed tomography study. Neuroimage 35:1409-1423, 2007

5. Forrester KR, Stewart C, Tulip J, Leonard C, Bray RC: Comparison of laser speckle and laser Doppler perfusion imaging: measurement in human skin and rabbit articular tissue. Med Biol Eng Comput 40:687-697, 2002

6. Fujii M, Hara H, Meng W, Vonsattel JP, Huang Z, Moskowitz MA: Strain-related differences in susceptibility to transient forebrain ischemia in SV-129 and C57black/6 mice. Stroke 28:1805-1811, 1997

7. Ginsberg MD, Busto R: Rodent models of cerebral ischemia. Stroke 20:1627-1642, 1989

8. Kawahara N, Kawai K, Toyoda T, Nakatomi H, Furuya K, Kirino T: Cardiac arrest cerebral ischemia model in mice failed to cause delayed neuronal death in the hippocampus. Neurosci Lett 322:91-94, 2002

9. Keskintepe L, Norris K, Pacholczyk G, Dederscheck SM, Eroglu A: Derivation and comparison of C57BL/6 embryonic stem cells to a widely used 129 embryonic stem cell line. Transgenic Res 16:751-758, 2007

10. Kirino T: Delayed neuronal death in the gerbil hippocampus following ischemia. Brain Res 239:57-69, 1982

11. Kitagawa K, Matsumoto M, Yang G, Mabuchi T, Yagita Y, Hori M, et al: Cerebral ischemia after bilateral carotid artery occlusion and intraluminal suture occlusion in mice: evaluation of the patency of the posterior communicating artery. $\mathbf{J}$ Cereb Blood Flow Metab 18:570-579, 1998

12. Kreisman NR, Soliman S, Gozal D: Regional differences in hypoxic depolarization and swelling in hippocampal slices. J Neurophysiol 83:1031-1038, 2000

13. Murakami K, Kondo T, Kawase M, Chan PH: The development of a new mouse model of global ischemia: focus on the relationships between ischemia duration, anesthesia, cerebral vasculature, and neuronal injury following global ischemia in mice. Brain Res 780:304-310, 1998

14. Olsson T, Wieloch T, Smith ML: Brain damage in a mouse model of global cerebral ischemia. Effect of NMDA receptor blockade. Brain Res 982:260-269, 2003

15. Onken M, Berger S, Kristian T: Simple model of forebrain ischemia in mouse. J Neurosci Methods 204:254-261, 2012

16. Panahian N, Yoshida T, Huang PL, Hedley-Whyte ET, Dalkara T, Fishman MC, et al: Attenuated hippocampal damage after global cerebral ischemia in mice mutant in neuronal nitric oxide synthase. Neuroscience 72:343-354, 1996

17. Pegorini S, Braida D, Verzoni C, Guerini-Rocco C, Consalez GG, Croci L, et al: Capsaicin exhibits neuroprotective effects in a model of transient global cerebral ischemia in Mongolian gerbils. Br J Pharmacol 144:727-735, 2005

18. Pulsinelli WA, Brierley JB: A new model of bilateral hemispheric ischemia in the unanesthetized rat. Stroke 10:267272, 1979

19. Pulsinelli WA, Brierley JB, Plum F: Temporal profile of neuronal damage in a model of transient forebrain ischemia. Ann Neurol 11:491-498, 1982

20. Sheng H, Laskowitz DT, Pearlstein RD, Warner DS: Characterization of a recovery global cerebral ischemia model in the mouse. J Neurosci Methods 88:103-109, 1999

21. Smith ML, Auer RN, Siesjö BK: The density and distribution of ischemic brain injury in the rat following 2-10 min of forebrain ischemia. Acta Neuropathol 64:319-332, 1984

22. Tsuchiya D, Hong S, Kayama T, Panter SS, Weinstein PR: Effect of suture size and carotid clip application upon blood flow and infarct volume after permanent and temporary mid- 
dle cerebral artery occlusion in mice. Brain Res 970:131139,2003

23. Türeyen K, Vemuganti R, Sailor KA, Dempsey RJ: Ideal suture diameter is critical for consistent middle cerebral artery occlusion in mice. Neurosurgery 56 (1 Suppl):196-200, 2005

24. Wei G, Doré S: Importance of normothermia control in investigating delayed neuronal injury in a mouse global ischemia model. J Neurosci Methods 185:230-235, 2010

25. Wellons JC III, Sheng H, Laskowitz DT, Mackensen GB, Pearlstein RD, Warner DS: A comparison of strain-related susceptibility in two murine recovery models of global cerebral ischemia. Brain Res 868:14-21, 2000

26. Yang G, Kitagawa K, Matsushita K, Mabuchi T, Yagita Y, Yanagihara T, et al: C57BL/6 strain is most susceptible to cerebral ischemia following bilateral common carotid occlusion among seven mouse strains: selective neuronal death in the murine transient forebrain ischemia. Brain Res 752:209_ 218, 1997

27. Yonekura I, Kawahara N, Nakatomi H, Furuya K, Kirino T: A model of global cerebral ischemia in C57 BL/6 mice. $\mathbf{J}$ Cereb Blood Flow Metab 24:151-158, 2004

28. Yoshioka H, Niizuma K, Katsu M, Sakata H, Okami N, Chan $\mathrm{PH}$ : Consistent injury to medium spiny neurons and white matter in the mouse striatum after prolonged transient global cerebral ischemia. J Neurotrauma 28:649-660, 2011
29. Zhen G, Doré S: Optimized protocol to reduce variable outcomes for the bilateral common carotid artery occlusion model in mice. J Neurosci Methods 166:73-80, 2007

\section{Author Contributions}

Acquisition of data: Nishijima. Analysis and interpretation of data: Niizuma, Nishijima. Drafting the article: Nishijima. Critically revising the article: Niizuma, Fujimura, Shimizu, Tominaga. Reviewed submitted version of manuscript: Niizuma, Akamatsu, Shimizu. Approved the final version of the manuscript on behalf of all authors: Niizuma. Study supervision: Niizuma.

\section{Supplemental Information}

\section{Current Affiliation}

Dr. Shimizu: Department of Neurosurgery, Akita University School of Medicine, Akita, Japan.

\section{Correspondence}

Kuniyasu Niizuma, Department of Neurosurgery, Tohoku University Graduate School of Medicine, 1-1 Seiryo-machi, Aoba-ku, Sendai, Miyagi 980-8574, Japan.email: niizuma@nsg. med.tohoku.ac.jp. 